\title{
Performance Optimization of Au-Free Lateral AlGaN/GaN Schottky Barrier Diode With Gated Edge Termination on 200-mm Silicon Substrate
}

\author{
Jie Hu, Student Member, IEEE, Steve Stoffels, Silvia Lenci, Benoit Bakeroot, Brice De Jaeger, \\ Marleen Van Hove, Nicolò Ronchi, Rafael Venegas, Hu Liang, Ming Zhao, \\ Guido Groeseneken, Fellow, IEEE, and Stefaan Decoutere
}

\begin{abstract}
In this paper, a further leakage reduction of AlGaN/GaN Schottky barrier diodes with gated edge termination (GET-SBDs) has been achieved by optimizing the physical vapor deposited $\mathrm{TiN}$ as the anode metal without severe degradation of ON-state characteristics. The optimized GET-SBD multifinger power diodes with $10 \mathrm{~mm}$ anode width deliver $\sim 4 \mathrm{~A}$ at $2 \mathrm{~V}$ and show a median leakage of $1.3 \mu \mathrm{A}$ at $25{ }^{\circ} \mathrm{C}$ and $3.8 \mu \mathrm{A}$ at $150{ }^{\circ} \mathrm{C}$ measured at a reverse voltage of $-200 \mathrm{~V}$. The temperature-dependent leakage of $\mathrm{Si}, \mathrm{SiC}$, and our $\mathrm{GaN}$ power diodes has been compared. The breakdown voltage (BV) of GET-SBDs was evaluated by the variation of anode-to-cathode spacing $\left(L_{\mathrm{AC}}\right)$ and the length of field plate. We observed a saturated $\mathrm{BV}$ of $\sim 600 \mathrm{~V}$ for the GET-SBDs with $L_{\mathrm{AC}}$ larger than $5 \mu \mathrm{m}$. The GET-SBD breakdown mechanism is shown to be determined by the parasitic vertical leakage current through the $2.8 \mu \mathrm{m}$-thick buffer layers measured with a grounding substrate. Furthermore, we show that the forward voltage of GET-SBDs can be improved by shrinking the lateral dimension of the edge termination due to reduced series resistance. The leakage current shows no dependence on the layout dimension $L_{G}$ (from 2 to $0.75 \mu \mathrm{m}$ ) and remains at a value of $\sim 10 \mathrm{nA} / \mathrm{mm}$. The optimized Au-free GET-SBD with low leakage current and improved forward voltage competes with high-performance lateral AIGaN/GaN SBDs reported in the literature.
\end{abstract}

Index Terms-200-mm substrate, AlGaN/GaN, Au-free, edge termination, leakage reduction, Schottky barrier diode (SBD), TiN.

\section{INTRODUCTION}

$\mathbf{O}$ VER the last few years, electronic devices based on $\mathrm{AlGaN} / \mathrm{GaN}$ heterojunction have emerged as promising

Manuscript received October 23, 2015; revised December 15, 2015; accepted January 5, 2016. Date of publication January 20, 2016; date of current version February 23, 2016. This work was supported by the imec's Industrial Affiliation Program through the GaN Project. The review of this paper was arranged by Editor W. Tsai.

J. Hu and G. Groeseneken are with imec, Leuven 3001, Belgium, and also with the Department of Electrical Engineering-Microelectronics and Sensors, KU Leuven, Leuven 3001, Belgium (e-mail: jie.hu@imec.be; guido.groeseneken@imec.be).

S. Stoffels, S. Lenci, B. De Jaeger, M. Van Hove, N. Ronchi, R. Venegas, H. Liang, M. Zhao, and S. Decoutere are with imec, Leuven 3001, Belgium (e-mail: steve.stoffels@imec.be; silvia.lenci@imec.be; brice.dejaeger@ imec.be; marleen.vanhove@imec.be; nicolo.ronchi@imec.be; rafael. venegas@imec.be; hu.liang@imec.be; ming.zhao@imec.be; stefaan. decoutere@imec.be).

B. Bakeroot is with imec, Leuven 3001, Belgium, and also with the Centre for Microsystems Technology, Department of Electronics and Information Systems, Ghent University, Ghent 9052, Belgium (e-mail: benoit.bakeroot@imec.be).

Color versions of one or more of the figures in this paper are available online at http://ieeexplore.iee.org.

Digital Object Identifier 10.1109/TED.2016.2515566 candidates for high-power switching applications due to the superior material properties compared with conventional silicon [1]-[5]. The wide bandgap nature of GaN-based electronic devices and the high mobility and electron density of the 2-D electron gas (2DEG) at the $\mathrm{AlGaN} / \mathrm{GaN}$ heterointerface lead to a high-power figure-of-merit (FOM) $\mathrm{BV}^{2} / R_{\mathrm{ON}, \mathrm{SP}}$ and enable power conversion with higher efficiency [6]. To enable low-cost production of GaN-based devices, many research groups have been developing $\mathrm{AlGaN} / \mathrm{GaN}$ epi-layers on large-area silicon substrates [7]. Recently, the fabrication of high-performance $\mathrm{AlGaN} / \mathrm{GaN}$ high electron mobility transistors and Schottky barrier diodes (SBDs) with a Au-free CMOS-compatible process flow on a 200-mm Si substrate has been a major breakthrough for the realization of efficient high-power devices at low manufacturing cost [4], [8].

In efficient power converters, the GaN-based SBD is required to have a low leakage current in the OFF-state and a low forward voltage drop in the ON-state, thus minimal static power loss of the diode can be obtained [9]. Partial or full AlGaN barrier recess in the anode region has been reported to increase the forward current and lower the forward voltage drop [3], [9]. Furthermore, AlGaN/GaN SBDs with gated ohmic anode structure have been proposed to achieve low turn-ON voltages [10]. Various designs of field-plates and edge terminations in lateral and vertical GaN-based SBDs have been reported to suppress the OFF-state leakage current [9], [11], [12]. Matioli et al. [13] presented a lateral $\mathrm{AlGaN} / \mathrm{GaN}$ SBD with a 3-D anode structure, where an integration of a trigate MOS structure with a Schottky junction is designed, demonstrating an ultralow leakage of $260 \mathrm{pA} / \mathrm{mm}$ and a turn-ON voltage of $0.85 \mathrm{~V}$. Among those promising $\mathrm{AlGaN} / \mathrm{GaN}$ SBD architectures, typical high workfunction $\left(\phi_{m}\right) \mathrm{Ni} / \mathrm{Au}$ or Pt-based metals were used for the fabrication of the Schottky contacts [3], [6], [10], [12], [13]. This hinders the high-volume production of those highperformance devices in existing CMOS-compatible processing lines because of their limited dry-etch capability [14].

In [4], we have demonstrated an architecture of the $\mathrm{AlGaN} / \mathrm{GaN}$ SBD with gated edge termination (GET-SBD) in Au-free technology and obtained significant diode leakage reduction and good ON-state characteristics. The embedded edge termination in the anode trench enables the reduction of the peak $E$-field at the anode edge; thus, a low leakage current was obtained in AlGaN/GaN SBDs [9]. Since the 
Schottky barrier height $\left(\mathrm{SBH} \phi_{B}\right)$ is an important parameter determining the diode characteristics, the selection of high work-function Au-free metals for anode electrode can potentially improve the OFF-state characteristics and optimize the performance of the AlGaN/GaN SBDs [15].

In this paper, we extensively study the impact of anode metal and layout parameters on the diode's OFF- and ON-state characteristics. We used two different types of TiN, a standard physical vapor deposited (PVD) TiN and a PVD TiN with ionized metal plasma source (IMP TiN), as the anode metal in intimate contact with the AlGaN barrier. The IMP TiN showed a high- $\phi_{m}$, which allows a further reduction in the leakage current of the AlGaN/GaN GET-SBD. The leakage reduction by optimizing the TiN has been confirmed on small diodes and multifinger power diodes with total anode width of $10 \mathrm{~mm}$. The diode forward voltage is found to scale down with the reduction of the length of edge termination without sacrificing the leakage characteristics. The benchmarking of the diode leakage current and forward voltage shows that our optimized GET-SBDs demonstrate very competitive performances with those of reported lateral $\mathrm{AlGaN} / \mathrm{GaN}$ SBDs, which are mostly fabricated with $\mathrm{Ni} / \mathrm{Au}$-based technologies [3], [6], [10], [12], [13].

\section{EXPERIMENTAL Details}

\section{A. Epi-Structure and Device Processing}

$\mathrm{AlGaN} / \mathrm{GaN}$ heterostructure layers were grown on 200-mm silicon wafers by metalorganic chemical vapor deposition (MOCVD). The epi-stack consists of a 10-nm $\mathrm{Al}_{0.25} \mathrm{Ga}_{0.75} \mathrm{~N}$ barrier, a $150-\mathrm{nm} \mathrm{GaN}$ channel layer, a $2.8-\mu \mathrm{m}$ C-doped AlGaN buffer as the back-barrier, and a 200-nm AlN nucleation layer on p-type $\mathrm{Si}(111)$ substrates. The passivation capping layer of the wafers was $5 \mathrm{~nm}$ in situ MOCVD $\mathrm{Si}_{3} \mathrm{~N}_{4}$. The surface of the entire stack was then encapsulated by a $140-\mathrm{nm} \mathrm{Si} \mathrm{S}_{4}$ layer by means of rapid thermal chemical vapor deposition. The removal of the $\mathrm{Si}_{3} \mathrm{~N}_{4}$ passivation layer in the anode region was performed by $\mathrm{SF}_{6}$ dry-etching. The $\mathrm{AlGaN}$ barrier recess was performed with an atomic layer etching process. After recess, the 5-nm AlGaN barrier was left in the anode region. To form an edge termination, the $15-\mathrm{nm} \mathrm{Si} \mathrm{Si}_{4}$ layer was deposited inside the anode trench by means of plasma-enhanced atomic layer deposition. The center region was opened to allow the formation of a Schottky contact on the top of the AlGaN barrier. Before the deposition of the anode metal, an in situ $\mathrm{N}_{2}$ plasma cleaning treatment of the anode area was done to clean the AlGaN surface [16]. The Au-free anode metal stack is composed of $20-\mathrm{nm}$ TiN/20-nm Ti/250-nm Al/20-nm Ti/60-nm TiN. An Au-free metal stack of Ti/Al-based metals was deposited for the cathode contact formation, which was annealed at $550{ }^{\circ} \mathrm{C}$ [4]. A schematic of the $\mathrm{AlGaN} / \mathrm{GaN}$ SBD with gated edge termination (GET-SBD) is shown in Fig. 1(a). Two-level anode FPs are fabricated on the $\mathrm{Si}_{3} \mathrm{~N}_{4}$ passivation layers toward the cathode. The layout design of the GET-SBD is shown in Fig. 1(b). The Schottky contact length $\left(L_{\mathrm{SC}}\right)$, the anode finger width, and the anode-to-cathode distance $\left(L_{\mathrm{AC}}\right)$ were 6,100 , and $5 \mu \mathrm{m}$, respectively. A cross-sectional SEM image of the anode

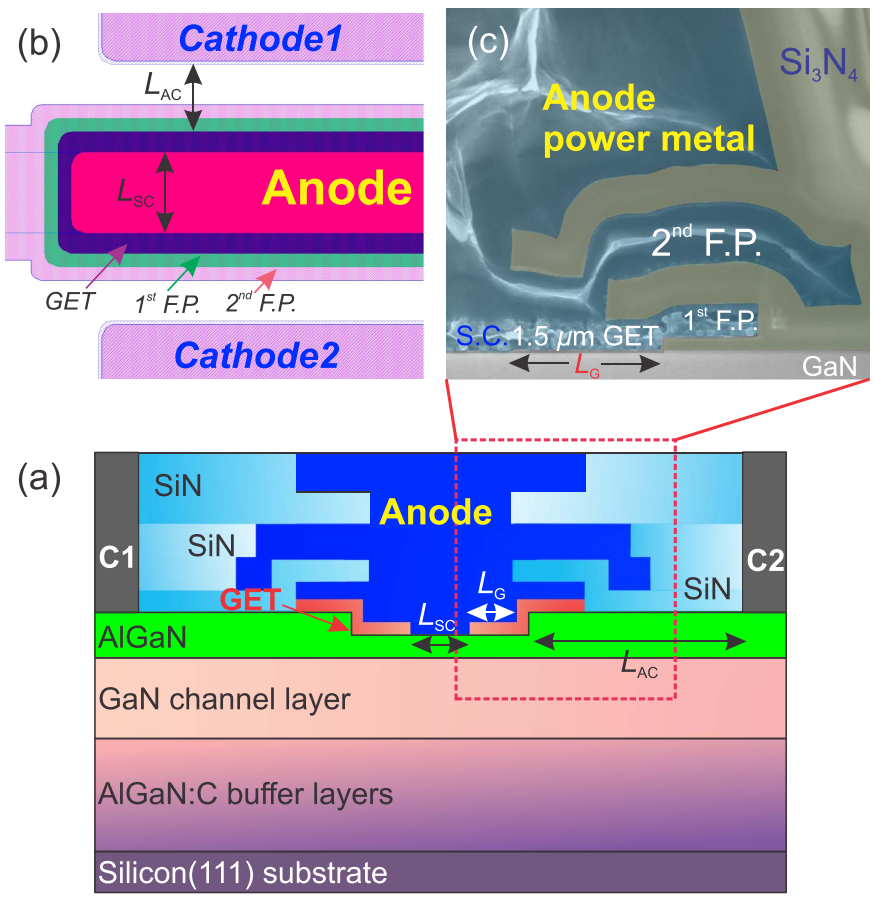

Fig. 1. (a) Schematic cross section of the AlGaN/GaN GET-SBD and two-level FPs. (b) Layout design of the GET-SBD. (c) Cross-sectional SEM image of the anode region in GET-SBD.

region in GET-SBD is shown in Fig. 1(c), presenting $L_{G}$ of $1.5-\mu \mathrm{m}$ gated edge termination (GET) and two-level anode metal FPs. The conventional $\mathrm{AlGaN} / \mathrm{GaN}$ SBD (without edge termination and without $\mathrm{AlGaN}$ barrier recess) was also processed as a reference, and the two-level FP structure was fabricated as well in the reference SBD.

\section{B. Optimization of TiN as Anode Metal}

Concerning the bottom 20-nm TiN layer, standard PVD TiN (at $23{ }^{\circ} \mathrm{C}$ ) and IMP PVD TiN (at $350{ }^{\circ} \mathrm{C}$ ) were used separately on two otherwise identical wafers. For the deposition of IMP TiN, an inductively coupled $\mathrm{Ar} / \mathrm{N}_{2}$ plasma was used to ionize the sputtered atoms at a relatively high temperature. The crystallographic orientation of PVD TiN and IMP TiN was examined by the grazing incidence X-ray diffraction (GIXRD) with spectra shown in Fig. 2(a). It was found that IMP TiN predominantly consists of (200) crystallites (a peak at $42.5^{\circ}$ ) compared with mixed grain orientations in standard PVD TiN [17]. The difference can be related to the difference in deposition temperature because metal tends to grow in a preferred orientation to obtain a stable crystal structure at high deposition temperatures [18]. The deposition details and material properties of these two TiN metals are further summarized in Table I. It was reported that the work-function of metals is dependent on the crystallographic orientation [19]. Capacitance-voltage $(C-V)$ measurements have been performed at $100 \mathrm{kHz}$ on conventional $\mathrm{AlGaN} / \mathrm{GaN}$ SBD and GET-SBD with IMP TiN and standard PVD TiN, respectively [in Fig. 2(b)]. A positive $V_{\mathrm{TH}}$ shift $(\sim 200 \mathrm{mV})$ is observed in the SBD and GET-SBD with IMP TiN compared with devices using PVD TiN, this indicates a higher $\phi_{m}$ for IMP TiN compared with standard PVD TiN. 

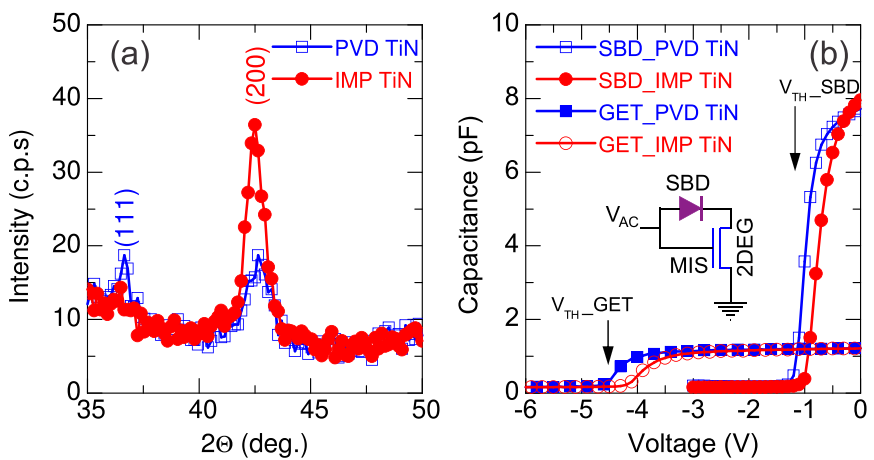

Fig. 2. (a) GIXRD spectra of the standard PVD TiN and the IMP TiN (b) Capacitance-voltage $(C-V)$ measurements on conventional AlGaN/GaN SBD and GET-SBD with IMP TiN and standard PVD TiN, respectively. The $V_{\mathrm{TH}}$ of SBD and GET-SBD is shifted positively with a value of $\sim 200 \mathrm{mV}$ with IMP TiN.

TABLE I

DEPOSITION DETAILS AND PROPERTIES OF STANDARD PVD TiN AND IMP TiN

\begin{tabular}{cccc}
\hline \hline & Temperature $\left({ }^{\circ} \mathrm{C}\right)$ & Thickness $(\mathrm{nm})$ & Crystal orientation \\
\hline PVD TiN & 350 & 20 & $(200)$ and $(111)$ \\
\hline IMP TiN & 23 & 20 & $(111)$ \\
\hline \hline
\end{tabular}
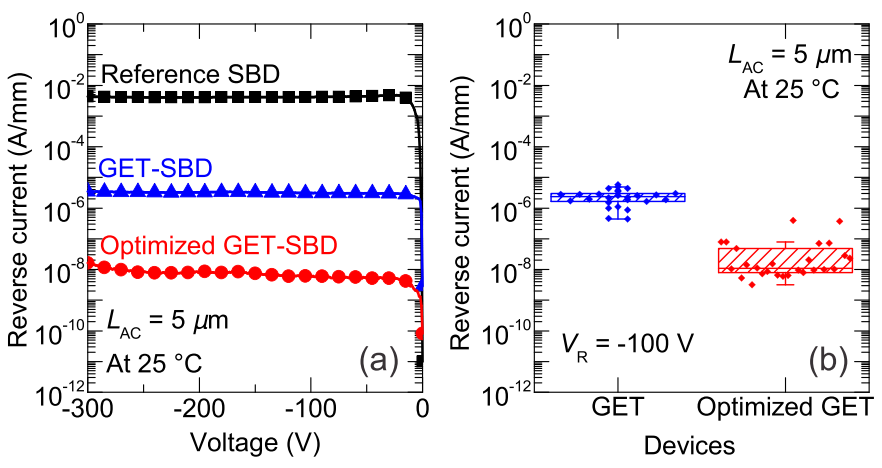

Fig. 3. (a) Typical OFF-state characteristics of the GET-SBD (labeled as GET-SBD1) and the one with optimized TiN anode metal (labeled as GET-SBD2) measured down to $V_{R}$ of $-300 \mathrm{~V}$, the leakage of a reference $\mathrm{AlGaN} / \mathrm{GaN}$ SBD is also shown. (b) Statistical plot of room-temperature leakage current (at $V_{R}$ of $-100 \mathrm{~V}$ ) for GET-SBD1 and GET-SBD2.

\section{RESUlts AND Discussion}

\section{A. Leakage Optimization With IMP TiN}

As displayed in Fig. 3(a), the leakage current of conventional SBD, GET-SBD with standard PVD TiN (labeled as GET-SBD1), and GET-SBD with IMP TiN (GET-SBD2) is measured $\left(\right.$ at $\left.25^{\circ} \mathrm{C}\right)$ down to a reverse voltage $\left(V_{R}\right)$ of $-300 \mathrm{~V}$. The conventional AlGaN/GaN SBD (with PVD TiN as the anode metal) suffers from high leakage due to electron tunneling through the $\mathrm{AlGaN}$ barrier layer in the lateral depletion region [9], though a $1-\mu \mathrm{m}$-long FP was fabricated on the 140-nm-thick $\mathrm{Si}_{3} \mathrm{~N}_{4}$ layer. In the GET-SBD, the edge termination (15-nm $\left.\mathrm{Si}_{3} \mathrm{~N}_{4}\right)$ pinches off the $2 \mathrm{DEG}$ channel effectively and redistributes the electric field in the vicinity of the Schottky contact [4], [9]. With the same anode metal of PVD TiN, GET-SBD1 shows three-order-of-magnitude reduction in leakage compared with the conventional SBD. When a
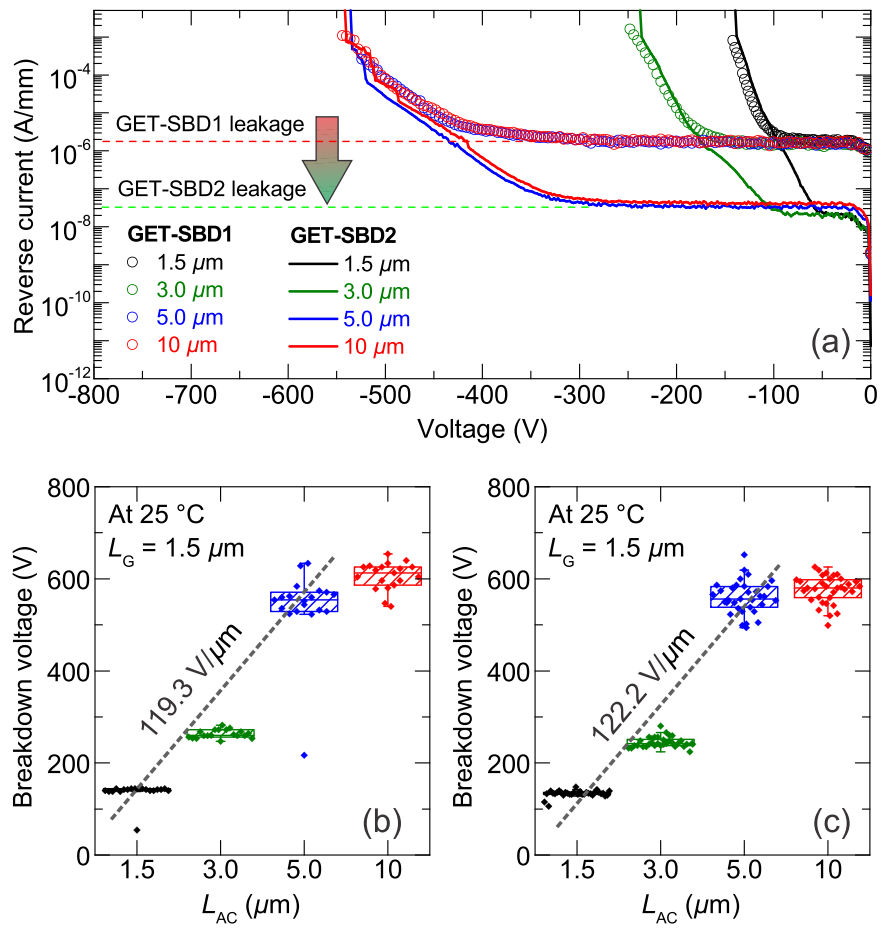

Fig. 4. (a) Typical leakage and breakdown characteristics of the GET-SBDs and optimized GET-SBDs with a variation of anode-to-cathode spacing $L_{\mathrm{AC}}$. The statistical plots of BV (at current level of $5 \mathrm{~mA} / \mathrm{mm}$ ) in GET-SBD1 and optimized GET-SBD2 with four different $L_{\mathrm{AC}}$ are shown in (b) and (c), respectively.

thicker $\mathrm{Si}_{3} \mathrm{~N}_{4}$ is used in the edge termination, the $V_{\mathrm{TH}}$ of the GET structure will shift in the negative direction. This can lead to an increase of the leakage. By combining a high- $\phi_{m}$ IMP TiN and an edge termination in GET-SBD2, a higher SBH and lower $E$-field can be obtained simultaneously, resulting in a stronger blocking capability for the leakage. This yields a 5 to 6 orders of magnitude leakage reduction compared with the conventional devices with PVD TiN. In Fig. 3(b), the statistical leakage value over the 200-mm wafers (at $V_{R}$ of $-100 \mathrm{~V}$ ) of GET-SBD1 and optimized GET-SBD2 with IMP TiN is shown. It confirms that the leakage current can be further reduced with a high $\phi_{m}$ IMP TiN as anode metal. The optimized GET-SBD2 shows a median leakage value of $10.8 \mathrm{nA} / \mathrm{mm}$ compared with $2.39 \mu \mathrm{A} / \mathrm{mm}$ in the case of GET-SBD1 with standard TiN as the anode metal.

To evaluate the breakdown voltage (BV), OFF-state measurements on both GET-SBD1 and optimized GET-SBD2 with a variation of anode-to-cathode spacing $L_{\mathrm{AC}}$ (from 1.5 to $10 \mu \mathrm{m})$ are performed. As presented in Fig. 4(a), the diode leakage current (prior to the diode breakdown or buffer breakdown) is independent of $L_{\mathrm{AC}}$ in both GET-SBDs due to the function of the gated edge termination. This observation is different from the results reported in [10] and [20], where a $L_{\mathrm{AC}}$-dependent leakage characteristics were shown. The difference in leakage current between the GET-SBD1 and the optimized GET-SBD2 is further shown in Fig. 4(a). The $\mathrm{BV}$ of these two GET-SBDs with identical $L_{\mathrm{AC}}$ appears to be the same, driven by the same breakdown mechanism. In Fig. 4(b) and (c), the statistical BV values (when the leakage reaches $5 \mathrm{~mA} / \mathrm{mm}$ ) of GET-SBD1 and GET-SBD2 with 

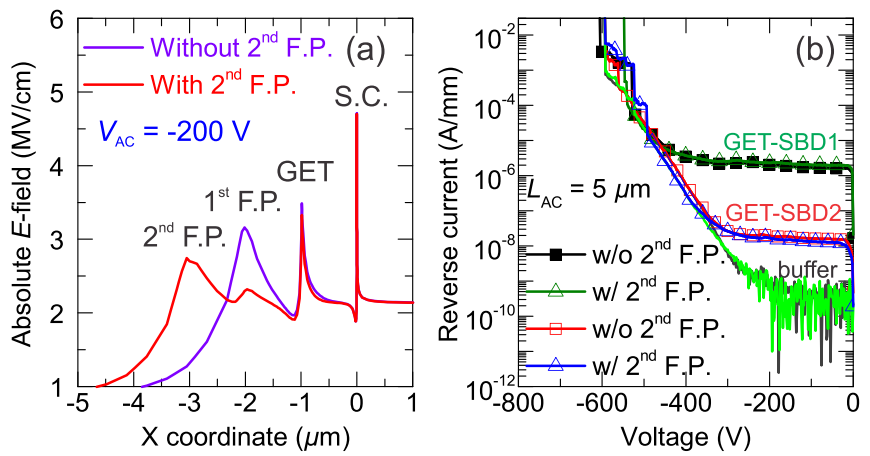

Fig. 5. (a) TCAD simulation of electric field distribution in $\mathrm{AlGaN}$ barrier $(0.5 \mathrm{~nm}$ away from the anode contact) at $-200 \mathrm{~V}$. (b) Breakdown characteristics of GET-SBD1 and GET-SBD2 with variation of the second FP, the buffer leakage currents were monitored during the measurements.

$L_{\mathrm{AC}}$ variations are shown. With the increase of $L_{\mathrm{AC}}$ dimension (from 1.5 to $5 \mu \mathrm{m}$ ), there is a linear increase $(\sim 120 \mathrm{~V} / \mu \mathrm{m}$ ) of the BV observed in both structures. This BV mechanism is triggered in the $\mathrm{AlGaN} / \mathrm{GaN}$ active layers in the vicinity of the anode contact, where a peak electric field is present. A further increase of $L_{\mathrm{AC}}$ dimension from 5 to $10 \mu \mathrm{m}$, however, does not lead to a further enhancement of BV. This is due to the buffer breakdown in the vertical direction, where the electric field increases vertically as the reverse voltage increases. For GET-SBD with $L_{\mathrm{AC}}$ of $5 \mu \mathrm{m}$, the diode shows excellent performance for $200-\mathrm{V}$ applications. Further buffer design and optimization are needed to boost the buffer BV allowing for higher voltage applications of the diode.

In Fig. 5(a), the electric field distribution in the AlGaN barrier $(0.5 \mathrm{~nm}$ away from the contact) was simulated in TCAD for GET-SBD with a variation of the second FP. The electric field is more uniformly distributed at the access region; however, the peak electric field at the Schottky contact (SC) does not change with an implementation of the second FP structure. In Fig. 5(b), the breakdown characteristics of GET-SBD1 and GET-SBD2 $\left(L_{\mathrm{AC}}\right.$ of $\left.5 \mu \mathrm{m}\right)$ are presented for devices with and without second FP. The buffer leakage currents were monitored simultaneously during the measurements. It is seen that the leakage value prior to breakdown is independent of the second FP for GET-SBD1 and GET-SBD2. This can be explained by the TCAD simulation in Fig. 5(a), which shows an unchanged peak $E$-field at the anode edge. When reverse voltage exceeds $400 \mathrm{~V}$, the buffer leakage becomes more dominant than the leakage of the diode. It further confirms that the buffer breakdown of the GET-SBD is the limiting factor for its high-voltage operations.

Multifinger GET-SBD power diodes with $10-\mathrm{mm}$ anode width [layout is shown in Fig. 6(a)] have been characterized with the substrate grounded. The temperature-dependent OFF-state characteristics of GET-SBD1 and GET-SBD2 are shown in Fig. 6(b). The statistical leakage current (at $V_{R}$ of $-200 \mathrm{~V}$ ) at $25{ }^{\circ} \mathrm{C}$ and $150{ }^{\circ} \mathrm{C}$ for GET-SBD1 and GET-SBD2 power diodes are presented in Fig. 6(c) and (d), respectively. These results show a good yield of the power diodes, and the optimized GET-SBD2 demonstrates a leakage current of $1.3 \mu \mathrm{A}$ at $25{ }^{\circ} \mathrm{C}$ and $3.8 \mu \mathrm{A}$ at $150{ }^{\circ} \mathrm{C}$ with very weak temperature dependence. This indicates that
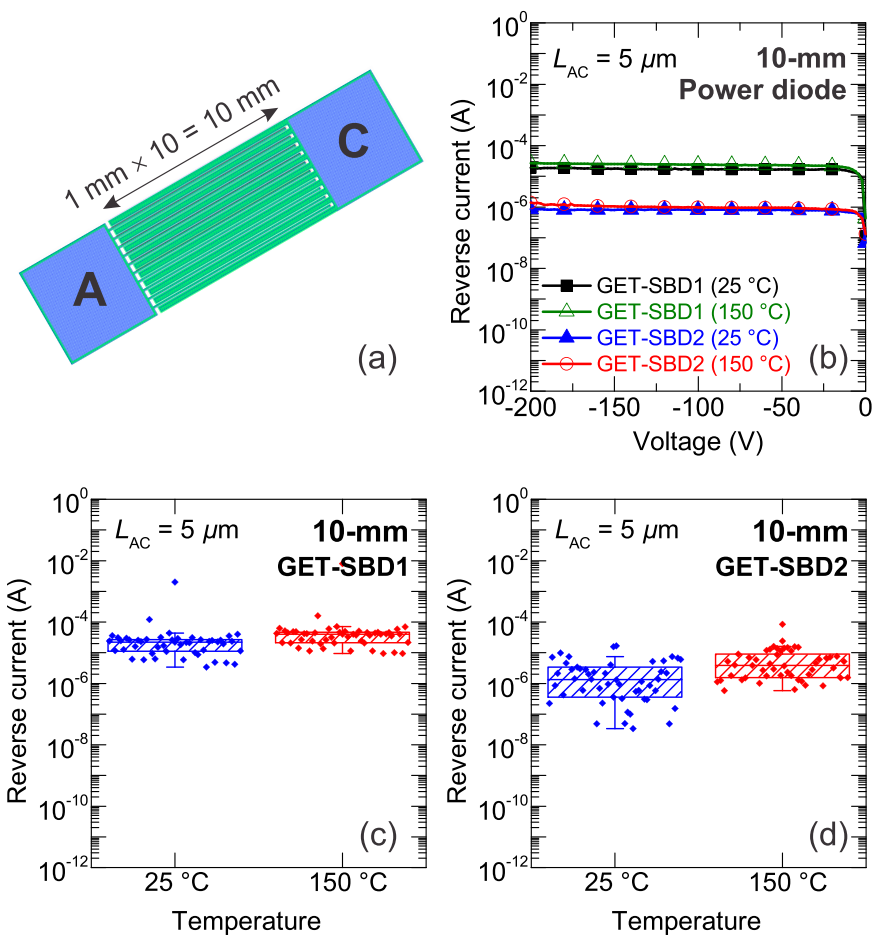

Fig. 6. (a) Layout of 10-mm multifinger GET-SBD. (b) Typical OFF-state characteristics of GET-SBD1 and GET-SBD2 power diodes measured at $25{ }^{\circ} \mathrm{C}$ and $150{ }^{\circ} \mathrm{C}$. The statistical leakage values of GET-SBD1 and GET-SBD2 (at $25^{\circ} \mathrm{C}$ and at $150^{\circ} \mathrm{C}$ ) are shown in (c) and (d), respectively.
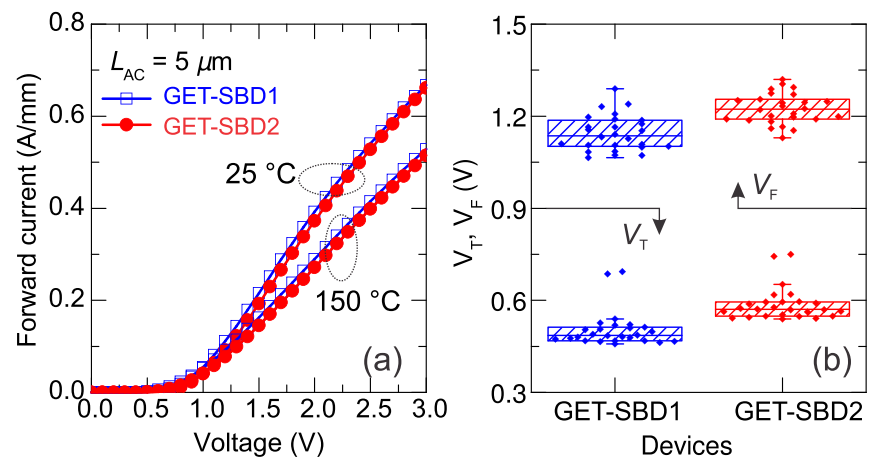

Fig. 7. (a) Typical forward characteristics of GET-SBD1 and GET-SBD2 at both $25{ }^{\circ} \mathrm{C}$ and $150{ }^{\circ} \mathrm{C}$. (b) Statistical plot of turn-ON voltage $V_{T}$ (at forward current of $1 \mathrm{~mA} / \mathrm{mm}$ ) and forward voltage $V_{F}$ (at forward current of $100 \mathrm{~mA} / \mathrm{mm}$ ) for GET-SBD1 and GET-SBD2 at $25{ }^{\circ} \mathrm{C}$.

our optimized GET-SBDs are promising for high-temperature and high-power applications where a low leakage current is required.

\section{B. Forward Current in GET-SBD With $P V D$ TiN and IMP TiN}

The typical forward characteristics of GET-SBD1 and GET-SBD2 at both $25{ }^{\circ} \mathrm{C}$ and $150{ }^{\circ} \mathrm{C}$ are presented in Fig. 7(a). The statistical values of turn-ON voltage $V_{T}$ (at forward current of $1 \mathrm{~mA} / \mathrm{mm} \mathrm{[6],} \mathrm{[10],} \mathrm{[20])} \mathrm{and} \mathrm{forward}$ voltage $V_{F}$ (at forward current of $100 \mathrm{~mA} / \mathrm{mm}$ ) for GET-SBD1 and GET-SBD2 are shown in Fig. 7(b). The forward voltage defined here combines the effect of the turn-ON voltage and the on-resistance of the diode, which can be a good operating 
TABLE II

Temperature-Dependent Leakage (at $V_{\mathrm{ac}}$ OF -200 V) Benchmarking of Si, SiC, and Lateral GaN Power Diodes

\begin{tabular}{cccccc}
\hline \hline Device & Forward voltage $\left(\right.$ at $\left.25{ }^{\circ} \mathrm{C}\right)$ at $1 \mathrm{~A}$ & $I_{\mathrm{OFF}}\left(25^{\circ} \mathrm{C}\right)$ & $I_{\mathrm{OFF}}\left(150{ }^{\circ} \mathrm{C}\right)$ & $I_{\mathrm{OFF}}\left(150{ }^{\circ} \mathrm{C}\right) / I_{\mathrm{OFF}}\left(25^{\circ} \mathrm{C}\right)$ & Substrate cost per cm ${ }^{2}[21]$ \\
\hline GaN Diode (This work) & 1.20 & 1.3 & 3.8 & 2.9 & $\sim \$ 0.08$ \\
\hline Si Diode (1N4935) & $1.05-1.20$ & 0.1 & 200 & 2000 & $\sim \$ 0.08$ \\
\hline SiC Diode (Ref. [22]) & 1.20 & 0.01 & 0.1 & 10 & $\sim \$ 6$ \\
\hline \hline
\end{tabular}
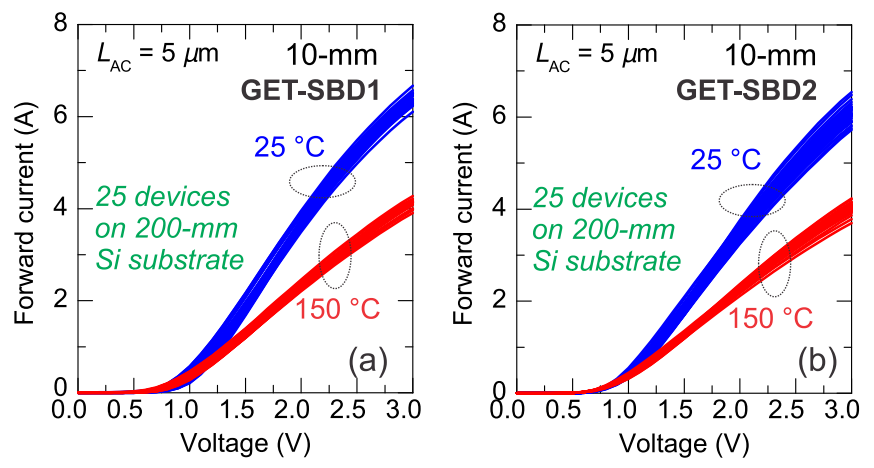

Fig. 8. Forward characteristics of (a) GET-SBD1 power diodes and (b) GET-SBD2 power diodes measured at $25{ }^{\circ} \mathrm{C}$ and $150{ }^{\circ} \mathrm{C}$. The results show high yield and good uniformity over $200-\mathrm{mm}$ silicon wafers.

point of the diode. The median values of $V_{F}$ for GET-SBD1 and GET-SBD2 are 1.14 and $1.22 \mathrm{~V}$, respectively. Though the anode metal IMP TiN has a higher $\phi_{m}$, no severe degradation of ON-state characteristics of GET-SBDs is observed. By using the formula describing the thermionic emission mechanism [23], the apparent SBH for GET-SBD1 and GET-SBD2 can be extracted to be 0.59 and $0.68 \mathrm{eV}$, respectively. The ideality factor values are 1.30 and 1.23 for GET-SBD1 and GET-SBD2, respectively. Besides the process of the injection of majority carriers over the Schottky barrier, there might be other current transport mechanisms (i.e., tunneling, trap-assisted tunneling, etc.) contributing to the ON-state currents [23].

In Fig. 8(a) and (b), the statistical forward characteristics (at $25{ }^{\circ} \mathrm{C}$ and $150{ }^{\circ} \mathrm{C}$ ) of 25 power diodes with $10-\mathrm{mm}$ anode width are presented for the GET-SBD1 and GET-SBD2 architectures. A high forward current of $\sim 4$ A (at $2 \mathrm{~V}$ ) at $25{ }^{\circ} \mathrm{C}$ for both GET-SBD1 and GET-SBD2 power diodes is demonstrated, together with a low leakage current shown in Fig. 6. This statistical evaluation confirms that the GET-SBD architecture with IMP TiN as the anode metal shows excellent performance with low leakage and high forward currents which has been demonstrated on 10-mm power diodes with high yield and good uniformity over a $200-\mathrm{mm}$ silicon wafers.

In Table II, a comparison of the temperature-dependent leakage (at $-200 \mathrm{~V}$ ) of $\mathrm{Si}, \mathrm{SiC}$ and $\mathrm{GaN}$ power diodes is shown. The forward voltage to deliver $1 \mathrm{~A}$ for three diodes is similar. The $\mathrm{SiC}$ diode shows the lowest leakage at both $25{ }^{\circ} \mathrm{C}$ and $150{ }^{\circ} \mathrm{C}$. However, the substrate cost is around 2 orders of magnitude higher than $\mathrm{Si}$ and GaN-on-Si diodes. A clear advantage of the GaN diode (GET-SBD2) compared with $\mathrm{Si}$ diode lies in its weak temperature-dependent leakage.

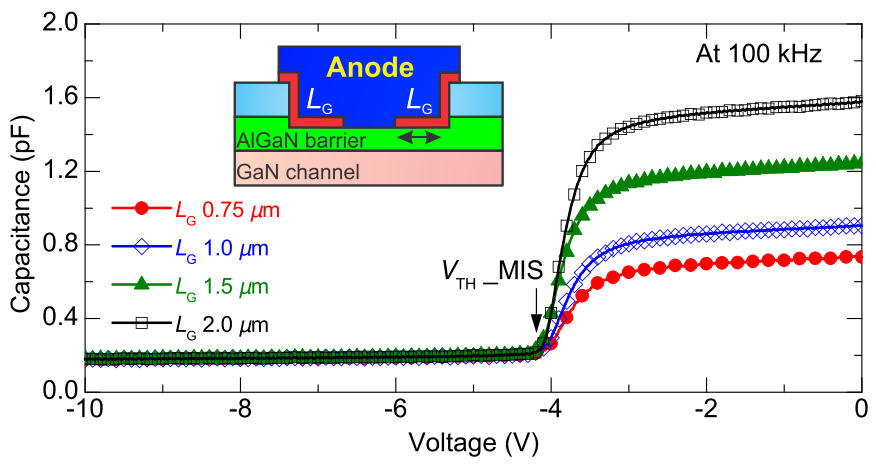

Fig. 9. $C-V$ measurements on GET-SBD2 with $L_{G}$ variations measured at $100 \mathrm{kHz}$. The inset schematic structure shows the anode region of the GET-SBD.
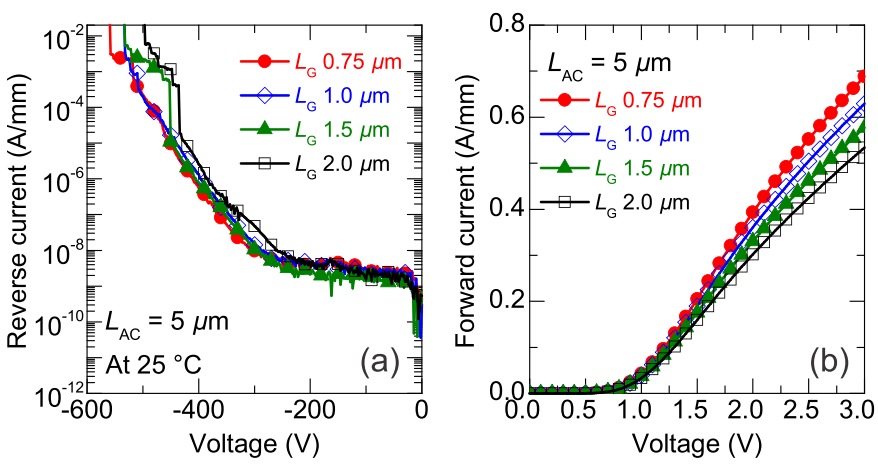

Fig. 10. Typical (a) OFF-state and (b) ON-state characteristics of the GET-SBDs with different $L_{G}$ dimensions.

Our Au-free GET-SBD power diode with low leakage and high forward currents demonstrates to be a promising candidate for low-cost and high-performance power electronics.

\section{C. $L_{G}$ Scaling}

The gated edge termination (with lateral dimension $L_{G}$ ) in GET-SBD allows for the redistribution of the electric field and the pinching-off of the 2DEG channel, thus low leakage current has been obtained. It is important to note that the dimension of $L_{G}$ contributes to the capacitance and the ON-state resistance. As shown in Fig. 9, the capacitancevoltage $(C-V)$ measurements have been performed at $100 \mathrm{kHz}$ on GET-SBD2 with different $L_{G}$. By scaling down $L_{G}$, the capacitance reduces linearly resulting in a more compact configuration. This can potentially reduce the cost and allows for the improvement of ON-state characteristics in GET-SBD2.

In Fig. 10(a) and (b), typical OFF-state and ON-state characteristics of GET-SBD2 (with $L_{\mathrm{AC}}$ of $5 \mu \mathrm{m}$ ) with different 

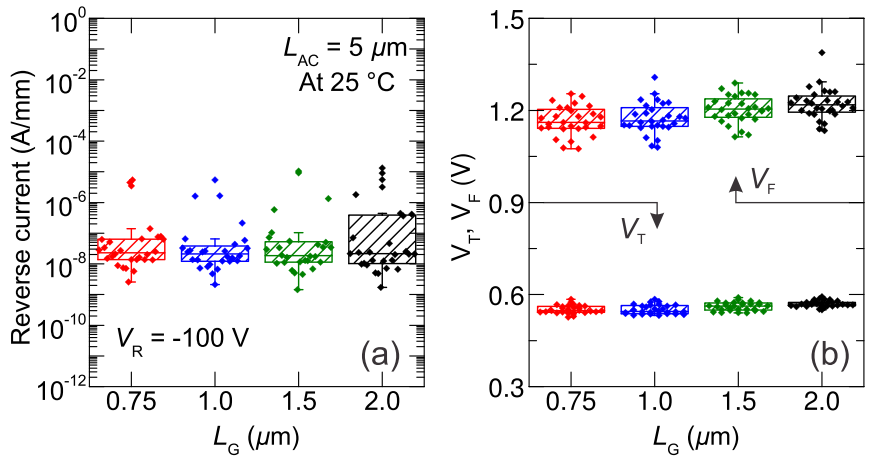

Fig. 11. (a) Statistical plot of leakage current at $V_{R}$ of -100 V. (b) Statistical values of $V_{T}$ and $V_{F}$ for GET-SBDs with a variation of $L_{G}$ dimensions.

$L_{G}$ are shown. The leakage characteristics of GET-SBDs show no degradation by scaling down $L_{G}$, while an improvement in forward characteristics is observed [shown in Fig. 10(b)] due to reduced series resistance. The statistics of leakage and ON-state voltages are presented in Fig. 11(a) and (b), respectively. By scaling down $L_{G}$ from 2 to $0.75 \mu \mathrm{m}$, the leakage current is invariant and maintains a low value. From the statistics of ON-state voltages, the turn-ON voltage does not change with $L_{G}$. A slight improvement of forward voltage is shown in Fig. 11(b). The median value of the forward voltage reduces from $1.22 \mathrm{~V}$ to $1.16 \mathrm{~V}$ by scaling down the $L_{G}$ from $2.0 \mu \mathrm{m}$ to $0.75 \mu \mathrm{m}$.

From the results shown in Fig. 11, a leakage current of $\sim 10.0 \mathrm{nA} / \mathrm{mm}$ and a forward voltage of $1.16 \mathrm{~V}$ have been achieved in GET-SBD2 (with IMP TiN) with $L_{G}$ of $0.75 \mu \mathrm{m}$. Shrinking $L_{G}$ allows for further optimization of the ON-state characteristics and a more compact design for further cost reduction of Au-free GaN-on-Si technology.

\section{Benchmarking of Leakage Versus Forward Voltage, $B V$ Versus $R_{O N, S P}$}

In this section, we firstly benchmark earlier reported $\mathrm{AlGaN} / \mathrm{GaN}$ lateral SBDs and this work (both $\mathrm{Au}$-free and $\mathrm{Ni} / \mathrm{Au}$-based technologies) in terms of leakage current (at $V_{R}$ of $-200 \mathrm{~V}$, leakage value from [13] was taken at $-127 \mathrm{~V}$ ) and the forward voltage (at forward current of $100 \mathrm{~mA} / \mathrm{mm}$ ). As shown in Fig. 12, most of the reported AlGaN/GaN SBDs were fabricated using $\mathrm{Ni} / \mathrm{Au}$ or Pt-based anode metal stacks, which are not compatible with the Si-based processing lines. Our Au-free AlGaN/GaN GET-SBDs with the optimized TiN metal show low leakage current of $\sim 10 \mathrm{nA} / \mathrm{mm}$, which shows competitive performance over most reported data. By scaling down the $L_{G}$ parameter, the forward voltage can be further reduced without degradation of OFF-state characteristics. This work, to the best of our knowledge, leads to lateral $\mathrm{AlGaN} / \mathrm{GaN}$ SBDs with the state-of-the-art performance in terms of combined low leakage and low forward voltage. Moreover, excellent characteristics have been demonstrated on power diodes with good yield and uniformity (at both $25^{\circ} \mathrm{C}$ and $150{ }^{\circ} \mathrm{C}$ ) on $200-\mathrm{mm}$ silicon substrate and realized by fabrication in a CMOS-compatible process line.

In Fig. 13, the benchmark plot of $\mathrm{BV}$ versus $R_{\mathrm{ON}, \mathrm{SP}}$ for lateral $\mathrm{AlGaN} / \mathrm{GaN}$ SBDs on $\mathrm{Si}$ and $\mathrm{SiC}$ substrates is shown.

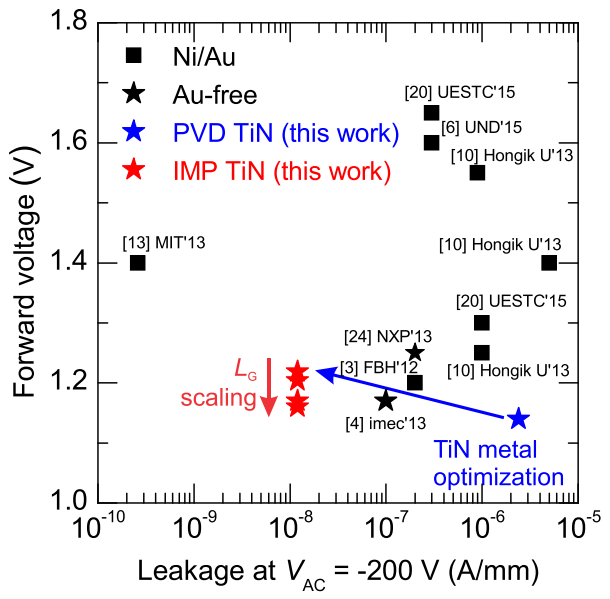

Fig. 12. Benchmarking the leakage current and forward voltage of lateral AlGaN/GaN Schottky diodes with $\mathrm{Ni} / \mathrm{Au}$ and $\mathrm{Au}$-free technology.

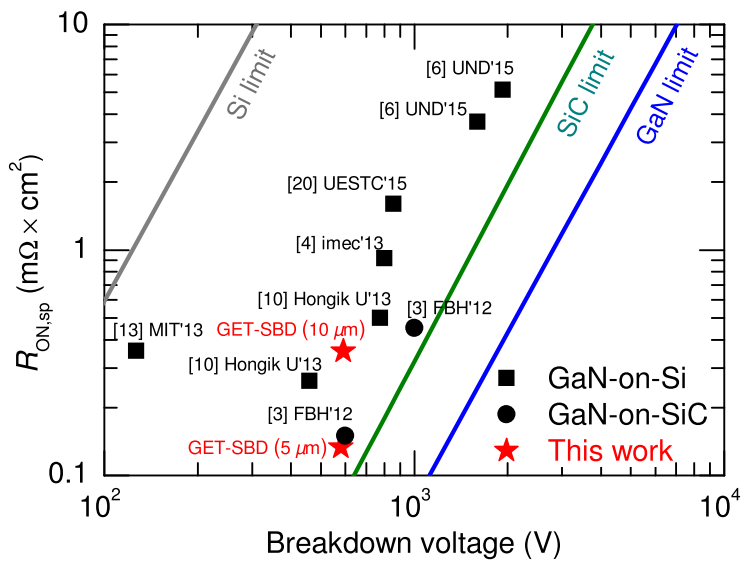

Fig. 13. Benchmarking the $\mathrm{BV}$ and specific on-resistance of lateral AlGaN/GaN on silicon and $\mathrm{SiC}$ substrate.

The high-performance GET-SBD with $L_{\mathrm{AC}}$ of $5 \mu \mathrm{m}$ demonstrates a power FOM $\left(\mathrm{BV}^{2} / R_{\mathrm{ON}, \mathrm{SP}}\right)$ as high as $2.5 \mathrm{GW} / \mathrm{cm}^{2}$. However, GET-SBD with $L_{\mathrm{AC}}$ of $10 \mu \mathrm{m}$ did not show further increase of $\mathrm{BV}$ due to the limitation from the buffer.

\section{CONCLUSION}

This paper optimizes the leakage current of Au-free AlGaN/GaN GET-SBDs by using IMP TiN with a high work-function. A further improvement of the forward voltage is achieved by scaling down the layout parameter $L_{G}$ without degrading the leakage characteristics, thus a more compact design can be realized for further cost reduction. Highperformance AlGaN/GaN GET-SBDs have been demonstrated with 10-mm power diodes, showing high yield and good uniformity with 200-mm silicon substrates. Our GaN power diodes show weak temperature-dependent leakage compared with $\mathrm{Si}$ and $\mathrm{SiC}$ vertical diodes. The benchmarking of lateral AlGaN/GaN SBDs shows that our optimized Au-free 200-V AlGaN/GaN GET-SBDs are competitive in terms of low leakage current and low forward voltage in comparison with reported data from the literature. To boost the BV of the GET-SBD and allow for higher voltage operation, the design of a more resistive buffer is required. 


\section{ACKNOWLEDGMENT}

The authors would like to thank H. Tielens for the GIXRD measurements, F. Vleugels for the SEM image analysis, and Prof. E. Simoen and Dr. D. Dictus for the valuable discussions on the work.

\section{REFERENCES}

[1] S. L. Selvaraj, A. Watanabe, A. Wakejima, and T. Egawa, "1.4-kV breakdown voltage for $\mathrm{AlGaN} / \mathrm{GaN}$ high-electron-mobility transistors on silicon substrate," IEEE Electron Device Lett., vol. 33, no. 10, pp. 1375-1377, Oct. 2012.

[2] M. Van Hove et al., "CMOS process-compatible high-power low-leakage AlGaN/GaN MISHEMT on silicon," IEEE Electron Device Lett., vol. 33, no. 5, pp. 667-669, May 2012.

[3] E. Bahat-Treidel et al., "Fast-switching GaN-based lateral power Schottky barrier diodes with low onset voltage and strong reverse blocking," IEEE Electron Device Lett., vol. 33, no. 3, pp. 357-359, Mar. 2012.

[4] S. Lenci et al., "Au-free $\mathrm{AlGaN} / \mathrm{GaN}$ power diode on 8-in Si substrate with gated edge termination," IEEE Electron Device Lett., vol. 34, no. 8, pp. 1035-1037, Aug. 2013

[5] J. Hu et al., "Current transient spectroscopy for trapping analysis on Au-free AlGaN/GaN Schottky barrier diode," Appl. Phys. Lett., vol. 106, no. 8, p. 083502, 2015.

[6] M. Zhu et al., "1.9-kV AlGaN/GaN lateral Schottky barrier diodes on silicon," IEEE Electron Device Lett., vol. 36, no. 4, pp. 375-377, Apr. 2015.

[7] D. Christy et al., "Uniform growth of $\mathrm{AlGaN} / \mathrm{GaN}$ high electron mobility transistors on $200 \mathrm{~mm}$ silicon (111) substrate," Appl. Phys. Exp., vol. 6 , no. 2, p. 026501, 2013.

[8] B. De Jaeger et al., "Au-free CMOS-compatible AlGaN/GaN HEMT processing on $200 \mathrm{~mm}$ Si substrates," in Proc. IEEE 24th Int. Symp. Power Semiconductor Devices ICs (ISPSD), Jun. 2012, pp. 49-52.

[9] J. Hu, S. Lenci, S. Stoffels, B. De Jaeger, G. Groeseneken, and S. Decoutere, "Leakage-current reduction and improved on-state performance of Au-free AlGaN/GaN-on-Si Schottky diode by embedding the edge terminations in the anode region," Phys. Status Solidi C, vol. 11, nos. 3-4, pp. 862-865, 2014.

[10] J.-G. Lee, B.-R. Park, C.-H. Cho, K.-S. Seo, and H.-Y. Cha, "Low turnon voltage $\mathrm{AlGaN} / \mathrm{GaN}$-on-Si rectifier with gated ohmic anode," IEEE Electron Device Lett., vol. 34, no. 2, pp. 214-216, Feb. 2013.

[11] Y. Zhang et al., "GaN-on-Si vertical Schottky and p-n diodes," IEEE Electron Device Lett., vol. 35, no. 6, pp. 618-620, Jun. 2014.

[12] Q. Zhou et al., "Over $1.1 \mathrm{kV}$ breakdown low turn-on voltage GaN-on-Si power diode with MIS-gated hybrid anode," in Proc. IEEE 27th Int. Symp. Power Semiconductor Devices IC's (ISPSD), May 2015, pp. $369-372$.

[13] E. Matioli, B. Lu, and T. Palacios, "Ultralow leakage current AlGaN/GaN Schottky diodes with 3-D anode structure," IEEE Trans. Electron Devices, vol. 60, no. 10, pp. 3365-3370, Oct. 2013.

[14] D. W. Johnson et al., "Challenges of contact module integration for GaN-based devices in a Si-CMOS environment," J. Vac. Sci. Technol. B, vol. 32, no. 3, p. 030606, 2014.

[15] S. M. Sze and K. K. Ng, Physics of Semiconductor Devices. New York, NY, USA: Wiley, 2006.

[16] J. Hu et al., "Physical origin of current collapse in Au-free AlGaN/GaN Schottky barrier diodes," Microelectron. Rel., vol. 54, nos. 9-10, pp. 2196-2199, 2014.

[17] D. Dictus, D. Shamiryan, V. Paraschiv, W. Boullart, S. De Gendt, and S. Vanhaelemeersch, "Influence of crystallographic orientation on dry etch properties of TiN," J. Vac. Sci. Technol. B, vol. 24, no. 5, pp. 2472-2476, 2006.

[18] H. F. Dadgour, K. Endo, V. K. De, and K. Banerjee, "Grain-orientation induced work function variation in nanoscale metal-gate transistorsPart II: Implications for process, device, and circuit design," IEEE Trans. Electron Devices, vol. 57, no. 10, pp. 2515-2525, Oct. 2010.

[19] R. Smoluchowski, "Anisotropy of the electronic work function of metals," Phys. Rev., vol. 60, no. 9, pp. 661-674, 1941.

[20] Q. Zhou et al., "High reverse blocking and low onset voltage AlGaN/GaN-on-Si lateral power diode with MIS-gated hybrid anode," IEEE Electron Device Lett., vol. 36, no. 7, pp. 660-662, Jul. 2015.

[21] Y. Zhang et al., "Origin and control of OFF-state leakage current in GaN-on-Si vertical diodes," IEEE Trans. Electron Devices, vol. 62, no. 7, pp. 2155-2161, Jul. 2015.
[22] C. Miesner, R. Rupp, H. Kapels, M. Krach, and I. Zverev, "thinQ! Silicon carbide Schottky diodes: An SMPS circuit designer's dream comes true!" White Paper, 2001.

[23] E. Arslan, S. Altındal, S. Özçelik, and E. Ozbay, "Dislocation-governed current-transport mechanism in (Ni/Au)-AlGaN/AlN/GaN heterostructures," J. Appl. Phys., vol. 105, no. 2, p. 023705, 2009.

[24] J. J. T. M. Donkers et al., "600 V-900 V GaN-on-Si process technology for Schottky barrier diodes and power switches fabricated in a standard Si-production fab," in Proc. CS MANTECH Conf., 2013, pp. 259-262.

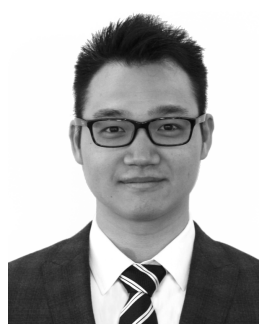

Jie Hu (S'14) received the M.Sc. (magna cum laude) degree in nanoscience and nanotechnology from the Katholieke Universiteit Leuven, Leuven, Belgium, in 2012, where he is currently pursuing the $\mathrm{Ph} . \mathrm{D}$. degree in electrical engineering.

He has been with the Power and Mixed Signal Technologies Group, imec, Leuven, since 2012. $\mathrm{He}$ is currently a Visiting Researcher with the Massachusetts Institute of Technology, Cambridge, MA, USA.

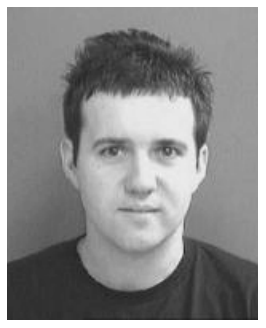

Steve Stoffels received the M.Sc. (magna cum laude) degree in applied physics from the University of Eindhoven, Eindhoven, The Netherlands, in 2001, and the Ph.D. degree from the Katholieke Universiteit Leuven, Leuven, Belgium, in 2010.

$\mathrm{He}$ is currently a Device Engineer with imec, Leuven, where he is involved in the research and development of high-power GaN technology. He is also acting as a Project Manager for funded projects.

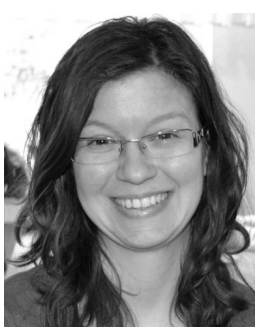

Silvia Lenci received the M.Sc. degree in electrical engineering from the University of Pisa, Pisa, Italy, in 2006, and the Ph.D. degree from the Department of Information Engineering, University of Pisa, in 2010.

She is currently with imec, Leuven, Belgium. Her current research interests include GaN-based high-power electronic devices and sensors.

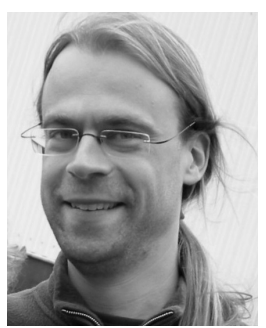

Benoit Bakeroot received the M.Sc. degree in physics and the Ph.D. degree in electrical engineering from Ghent University, Ghent, Belgium, in 1997 and 2004, respectively.

$\mathrm{He}$ joined imec, Leuven, Belgium, in 1998. $\mathrm{He}$ has been involved in GaN-on-Si devices since 2010. During his research career, he has used technology computer-aided design for the study of semiconductor devices. He is currently a part-time Assistant Professor with Ghent University.

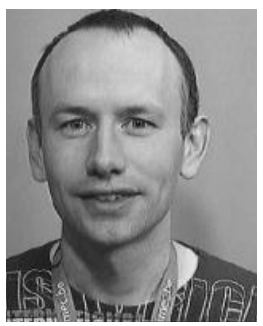

Brice De Jaeger received the B.S. and M.S. degrees in electrical engineering from Ghent University, Ghent, Belgium, in 1994 and 1997, respectively.

$\mathrm{He}$ has been a Research Engineer in different research departments with imec, Leuven, Belgium. $\mathrm{He}$ has collaborated in the technology development, integration, and characterization of interconnects and germanium CMOS transistors. His current research interests include $\mathrm{GaN}$ technology for power applications. 


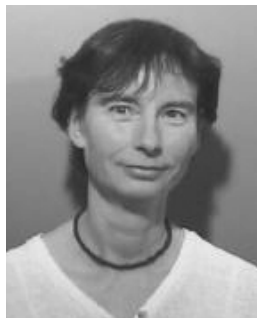

Marleen Van Hove received the M.S. degree in physics and the Ph.D. degree from the Catholic University of Leuven, Leuven, Belgium, in 1980 and 1985 , respectively.

She then joined imec, Leuven. She specialized in GaAs and InP III-V processing from 1986 to 1997. She was responsible for CMOS back-end integration at imec. In 2006, she returned to III-V research, where she is responsible for the development of high-power GaN-switching devices.

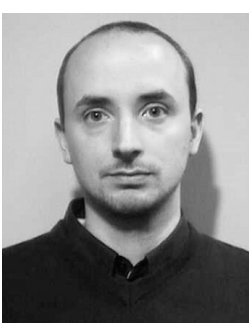

Nicolò Ronchi was born in Thiene, Italy. $\mathrm{He}$ received the Laurea degree in electronics engineering and the $\mathrm{Ph} . \mathrm{D}$. degree from the University of Padua, Padua, Italy, in 2008 and 2012, respectively.

$\mathrm{He}$ has been a Researcher with the Power and Mixed Signal Technologies Group, imec, Leuven, Belgium, since 2012. His current research interests include the characterization and reliability analysis of GaN-based semiconductor devices for highfrequency and power-switching applications.

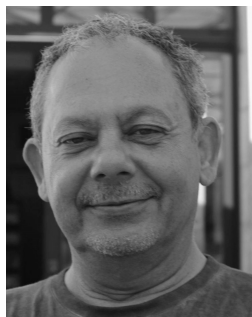

Rafael Venegas received the M.Sc. degree in physics from the University of Chile, Santiago, Chile, in 1976, and the Ph.D. degree in physics from University Joseph Fourier, Grenoble, France, in 1979 .

He was with the Chilean Atomic Energy Commission, Santiago, until he became an Associate Professor with the University of Santiago de Chile, Santiago, in 1985. In 2000, he joined the Mixed Signal Technologies Group, imec, Leuven, Belgium.

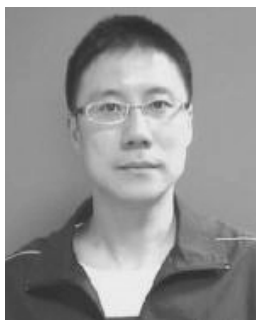

Hu Liang received the B.Sc. degree in physics from Beijing Normal University, Beijing, China, in 1996 and the M.Sc. and Ph.D. degrees in electronics and computer engineering from The Hong Kong University of Science and Technology, Hong Kong, in 2003 and 2007, respectively.

$\mathrm{He}$ has been with the Epitaxial Group, imec, Leuven, Belgium, since 2010, where he is currently a Senior Research Scientist.

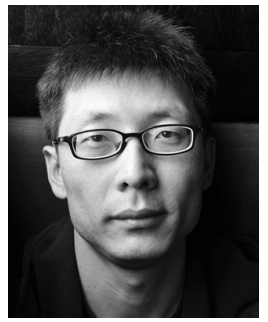

Ming Zhao received the M.Sc. degree in material engineering and the $\mathrm{Ph} . \mathrm{D}$. degree in material physics from Linköping University, Linköping, Sweden, in 2003 and 2008, respectively.

$\mathrm{He}$ joined imec, Leuven, Belgium, in 2008, as a Researcher, where he has been a Senior Researcher since 2012. His current research interests include epitaxy, material characterizations, and device physics of III-N and group IV materials.

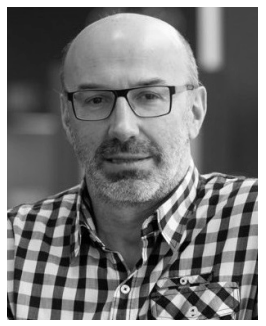

Guido Groeseneken (S'81-M'89-SM'95-F'05) received the M.Sc. degree in electrical engineering and the Ph.D. degree in applied sciences from the Katholieke Universiteit Leuven (KU Leuven), Leuven, Belgium, in 1980 and 1986, respectively.

He joined the Research and Development Laboratory, imec, Leuven, in 1987, where he became a fellow in 2007. Since 2001, he has been a Professor with the Department of Electrical Engineering, KU Leuven.

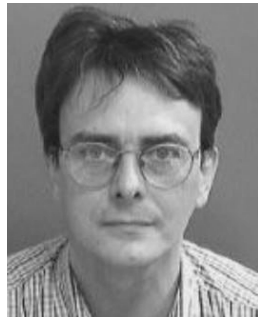

Stefaan Decoutere received the M.Sc. degree in electronic engineering and the Ph.D. degree from Katholieke Universiteit Leuven, Leuven, Belgium, in 1986 and 1992, respectively.

He has been with imec, Leuven, since 1987. In 1998, he became the Head of the Mixed Signal/RF Technology Group with imec. Since 2010, he manages the GaN Power Device technology development, and in 2015, he became the Director of the $\mathrm{GaN}$ technology program. 